\title{
Outdoor Self-Localization Method Based on Clustering and RSSI
}

\author{
Tong Wencan, Zhang Ling, Chen Pen, Huang Hongkun
}

School of Information engineering, Longyan University, Longyan, 364000

Keywords: Passive Localization, Wireless Sensor Network, Clustering, RSSI

\begin{abstract}
Focusing on the passive localization problem of the wireless sensor network (WSN) outdoor, an outdoor self-localization algorithm based on clustering and RSSI is proposed, which can localize the passive multi-targets in the wireless sensor network area. It uses the RSSI location model to realize the self-localization of sensor nodes, and then classifies the passive multi-targets based on clustering. Finally, the poly-gonization centroid algorithm is used to calculate the final position of the target. Simulation results prove that the proposed outdoor self-localization algorithm has low complexity and deviation, which is suitable for the passive multi-target localization.
\end{abstract}

\section{Introduction}

As the core technology in the era of Internet of things, wireless sensor network is widely used in various applications such as military, security, environmental monitoring, search and rescue, target tracking and other short distance wireless networking. It is based on the interaction between sensor nodes and environment, and nodes are usually randomly distributed in a certain monitoring area, so self-localization is the basis and premise of most application scenarios.

At present, the target location technology is mainly divided into two categories: active target location and passive target location. The essential difference is whether the target is equipped with devices to participate in the reception or transmission of wireless signals. Active positioning technique is usually required to carry target signal sending and receiving equipment, through the change of signal attenuation or phase to achieve the localization of [1]; passive positioning is not required to carry any target data transceiver equipment, the passive positioning and positioning to research the feasibility of accuracy improvement brings great difficulties to [2-3]. From the location algorithm, it can be divided into two categories based on distance measurement and non - range based. The location based on ranging can measure the distance or angle information between nodes, and calculate the location of nodes by using the three sides measurement, triangulation or maximum likelihood estimation. The commonly used ranging techniques include RSSI, TOA, TDOA and AOA [4]. The use of wireless signal strength location is characterized by high precision and small range positioning, but RSSI is relatively fragile, easily affected by environmental factors, we must establish in advance in the monitoring area position and the target position is received by the RSSI Association, passive target location is not suitable for outdoor large-scale regional environment. At the same time, the above location technology and location algorithm mostly aim at single target location, do not consider multi-target location problem, and the location complexity is high, so it is not suitable for random location in rain field [5].

In this paper, an outdoor adaptive target location algorithm based on clustering and RSSI is proposed. When the target node does not have the wireless signal transceiving function, it is possible to achieve a high-precision target node positioning. The algorithm realizes the location of passive random targets based on RSSI distance model, including node self-localization, aggregate hierarchical target clustering and three edge and multi weighted centroid algorithm.

\section{Algorithm Model}

In this algorithm, each sensor node does not require precise positioning of each node in the initialization phase relative position coordinates will adaptively establish each node in the sensor network, can meet the needs of practical application, and the algorithm to achieve the required 
hardware cost is low, the positioning process communication overhead is small, low power consumption. The algorithm uses the beacon node as the location of the target creature of the reference point.

\subsection{Wireless transmission loss model}

The accuracy of the RSSI location algorithm depends largely on the loss of the radio transmission road. The commonly used wireless propagation loss models include free space propagation model, logarithmic distance path loss model, Harbin model, logarithmic normal distribution model and so on [6].

Due to the influence of multipath, diffraction and obstacles, radio propagation loss will change with the theoretical value spread in free space under the actual environment. Therefore, the free space propagation model and the logarithmic normal distribution model are combined. Free space wireless transmission mode loss type is as follows:

$$
\text { loss }=32.44+10 k \lg d+10 k \lg f
$$

Among them, $d$ is the distance from the source of the distance, the unit is $\mathrm{km}$; $\mathrm{f}$ is the wireless frequency, the unit is MHZ; $k$ is the attenuation factor of the road strength.

The logarithmic normal distribution wireless propagation loss model is as follows:

$$
P L(d)=P L\left(d_{0}\right)+20 k \lg \left(d / d_{0}\right)+X \sigma
$$

Among them, $P L(d)$ is the path loss after distance $d$, the unit $\mathrm{dB}$ and $X \sigma$ is Gauss random distribution variables, the standard difference range is $4 \sim 10$, $\mathrm{k}$ is the road strength attenuation factor in 2 5. The value of $P L\left(d_{0}\right)$ can be replaced by the $\mathrm{d}=1 \mathrm{~m}$, which is replaced by the formula (1), and the obtained loss is the value o $P L\left(d_{0}\right)$. According to the formula (2), the signal intensity of each unknown node and beacon node can be obtained as follows:

RSSI = emission power + antenna gain - path loss $P L(d)$

Therefore, according to the above wireless transmission model, the beacon node receives the RSSI of any node, and the distance between the node and the beacon node can be obtained.

\subsection{Infrared range finding model}

Infrared ranging can be applied to targets whose temperature is higher than absolute zero. Electromagnetic radiation energy is an important parameter to detect target distance, and it depends on the target surface temperature $\mathrm{T}$ and wavelength. According to Planck's law, we can know the relationship between wavelength $\lambda$, temperature T, emissivity ${ }^{\varepsilon}$ and radiance $M_{\lambda}$.

$$
M_{\lambda}=\frac{\varepsilon \lambda c_{1}}{\lambda^{5}\left(\exp \left(C_{2} / \lambda T\right)-1\right)}, J_{e}=\frac{\varepsilon M_{\lambda} A}{\pi}=E_{e} d^{2}
$$

But the location principle is generally through a low frequency infrared, and then measuring the phase difference between the transmitted wave echo and the phase difference is calculated on the basis of $\Delta \varphi$, the echo time is $\Delta \mathrm{t}$ :

$$
\Delta t=(L / 2 \pi) \times \Delta \varphi
$$

Finally get the target distance. $\mathrm{L}$ is an infrared signal cycle.

\subsection{Location calculation}

In the field application scenario, the target node is a random target that can be induced by infrared sensors. It does not own wireless signals. The location information of the target node can 
only be obtained through the location information of the reference node and the detection range of the sensor [7]. By clustering and three edge and multilateral centroid algorithms, the target clustering and the final location coordinates of the target will be calculated. Each node in the wireless sensor network is called a reference node, which is composed of a wireless module and a sensor module. It can be further divided into beacon nodes and common nodes.

\subsubsection{Reference node self-positioning}

In this paper, three beacon nodes and three edge centroid algorithms are used to realize the self-positioning of other common nodes in the wireless sensor network. The location information of the beacon node can be manually configured with the aid of third party tools. Each common node, based on the RSSI value of other beacon nodes, establishes its own distance mapping with the beacon nodes, and sets up 2 sets.

Set of reference nodes reference set $=\left\{a_{0}, a_{1}, \ldots, a_{n-1}\right\}, \mathrm{n}$ is the total number of sensor nodes.

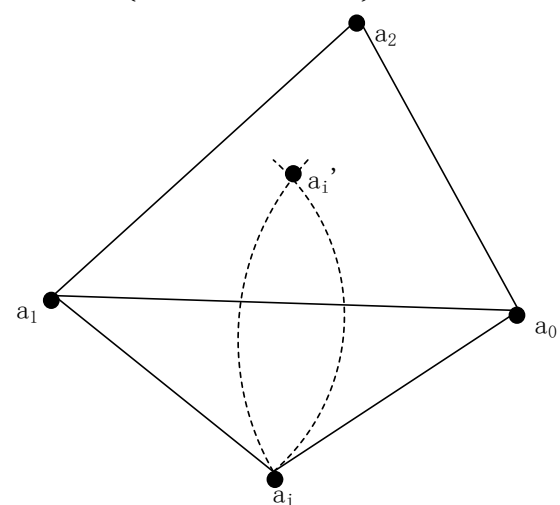

Fig.1 Common node location

\subsubsection{Target node location}

In the practical application environment, the location accuracy of target and the monitoring radius of monitoring nodes in the sensor network, the RSSI value of random components and the random distribution of wireless sensor network can be used to improve the positioning accuracy of the target by the centroid method or the maximum likelihood estimation method. But in practical application scenarios, there may be multiple targets at the same time [8]. Therefore, the use of centroid or maximum likelihood estimation can not guarantee the correctness of location. In this algorithm, the clustering algorithm is used to cluster the target, and the final location of different categories of targets is determined by the centroid method.

(a) Target clustering

There are a large number of clustering algorithms at present. For specific applications, the selection of clustering algorithms depends on the type of data and the purpose of clustering. The main clustering algorithms can be divided into the following categories: partition method, level method, density based method, grid based method and model-based method [9]. In this paper the cohesive hierarchical clustering algorithm is used to classify the target, and the distance metric method is the maximum distance metric method $d_{\max }\left(c_{i}, c_{j}\right)=\max _{p \in c_{i}}\left|p-p^{\prime}\right|$..There is $\mathrm{m}$ monitoring nodes to monitor the target, and the specific target clustering process is as follows:

Step1. The m nodes are regarded as a single class, and the maximum distance between 22 is calculated;

Step2. The maximum distance is less than the $2 r_{0}$ two merged into a new class;

Step3. Recalculate the distance between the new class and all classes;

Step4. Repeat step 2 and step 3, until all the inter class distance is not less than $2 r_{0}$.

After the processing of the target clustering algorithm, the number of the clusters or classes formed is the number of targets that need to be located at the present time.

(b) Target location calculation 
The target location is the process of calculating the final position coordinates of each target after the above target clustering. The calculation method is to calculate the centroid of each class of targets by using the weighted three side and the multilateral centroid method, so as to determine the final coordinates of the target [10-11]. Using the infrared range model in the previous article, the distance between all the monitoring nodes in the target class is calculated as the weight value of the location. M monitoring nodes through the cluster formed a number of classes, the number of nodes in the class with $n(n \geq 1)$.

If $\mathrm{n}=1$ is used, the coordinate monitoring node in the class of $\left(x_{i}, y_{i}\right)$ as the coordinate position of the target, namely position $_{\text {arg et }}=\left(x_{i}, y_{i}\right)$;

If $\mathrm{n}=2$, the target coordinates for mean two coordinate classes, namely: position $_{\text {target }}=\left(\frac{1}{2} \sum_{i=1}^{2} x_{i}, \frac{1}{2} \sum_{i=1}^{2} y_{i}\right)$;

If $\mathrm{n}=3, C_{n}^{3}$ triangle centroid is calculated by the weighted centroid method three edge, three edge centroid formula: $\left\{\begin{aligned} & x^{\prime}= \frac{\frac{x_{i}}{l_{i}+l_{j}}+\frac{x_{j}}{l_{j}+l_{k}}+\frac{x_{k}}{l_{i}+l_{k}}}{l_{i}+l_{j}}+\frac{1}{l_{j}+l_{k}}+\frac{1}{l_{i}+l_{k}} \\ & y^{\prime}= \frac{y_{i}}{l_{i}+l_{j}}+\frac{y_{j}}{l_{j}+l_{k}}+\frac{y_{k}}{l_{i}+l_{k}} \\ & \frac{1}{l_{i}+l_{j}}+\frac{1}{l_{j}+l_{k}}+\frac{1}{l_{i}+l_{k}}\end{aligned}\right.$ And then use the $C_{n}^{3}$ triangle centroid, then the centroid method for multilateral $\left\{x=\frac{1}{C_{n}^{8}} \sum_{i=1}^{C_{n}^{8}} x_{i}^{\prime}\left\{y=\frac{1}{C_{n}^{8}} \sum_{i=1}^{C_{n}^{8}} y_{i}^{\prime} \quad\right.\right.$ to calculate the target.

\section{Algorithmic Process}

The algorithm of outdoor adaptive target location based on clustering and RSSI is composed of three sub processes: self-positioning of reference nodes, target clustering and target location calculation. The algorithm can not only implement the passive location and tracking of the passive single target, but also realize the passive multi target location. The specific algorithm process is as follows [12]:

(1) The reference node self-localization, the establishment of referenece set $_{\text {set }}$ the reference node and reference node set distance $e_{\text {set }}$ and the reference node set position $_{\text {set }}=\left\{\left(x_{0}, y_{0}\right), \cdots\left(x_{n}, y_{n}\right)\right\}$.

(2) The target clustering algorithm is called, and the target class at the present time is constructed.

(3) Repeated calls to the target location calculation sub algorithm until all target classes are traversed.

(4) Repeat (2) and (3) calculate the position coordinates of the target at the next time.

\section{Simulation}

On the Matlab simulation platform, the simulation experiment is carried out on the proposed passive target positioning method. The simulation scenario is outdoor $50 \mathrm{~m} * 50 \mathrm{~m}$ area. The number of wireless sensor nodes (reference nodes) randomly distributed is 50 , and the sensor nodes cover the whole area of $25 \mathrm{~m} * 25 \mathrm{~m}$. The intersection density of nodes will affect the positioning accuracy. Therefore, the monitoring range of each node should be intersected with at least 2 other nodes' 
monitoring range to avoid node location coincidence. The relationship between the RSSI value and the distance is derived from the formula (1) and formula (2). The specific parameters of the simulation conditions are shown in Tab.1.

Tab.1 Simulation parameters

\begin{tabular}{ll}
\hline Parameter & Value \\
\hline Emission power/dBm & 0 \\
Antenna gain & 1 \\
Infrared monitoring radius/m & 3 \\
Attenuation factor & 3.4 \\
Gauss distribution random variable & 4 \\
Infrared wavelength $\lambda / \mathrm{m}$ & 0.02 \\
\hline
\end{tabular}

Fig.2 shows an analysis of the location results of a single target at 10 random locations of a sensor network at a given time. The average error is $1.18 \mathrm{~m}$, and the maximum error occurs mainly in the boundary of the monitoring area.

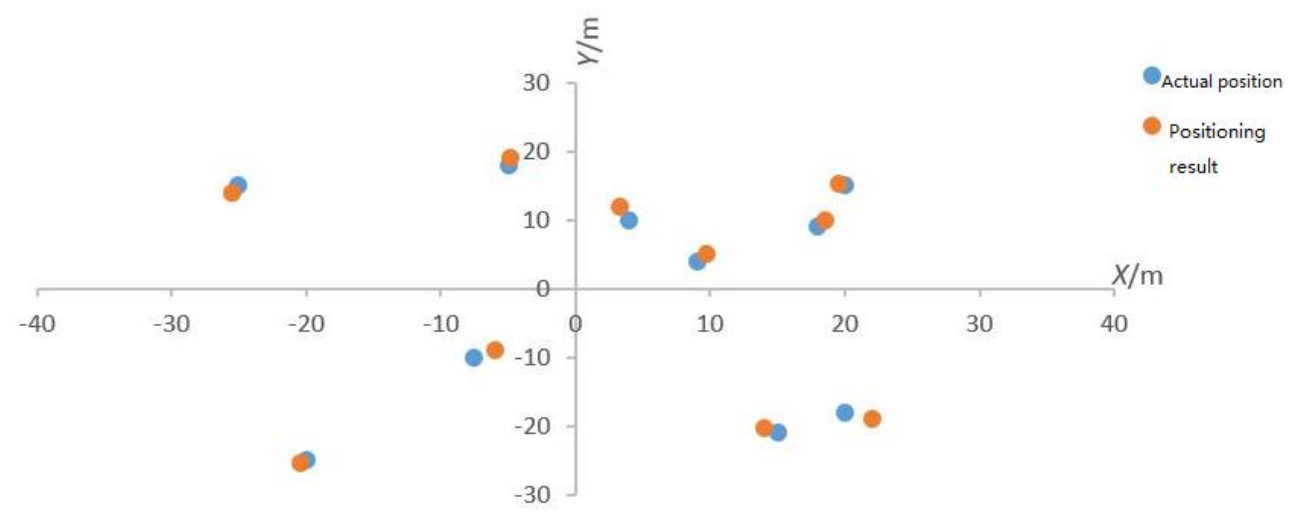

Fig. 2 The positioning result of a single target at 10 points at a given moment

Fig. 3 shows the location results of multiple targets in a certain period of time. The effect of positioning from the figure, the positioning results goals 1 and 2 in the sensor network layout in the actual position of track and method consistent with the trajectory that outdoor localization algorithm clustering and RSSI to meet the requirements of passive target positioning based on the proposed, but demanding accuracy in the case of wild passive positioning can achieve the goal, the better.

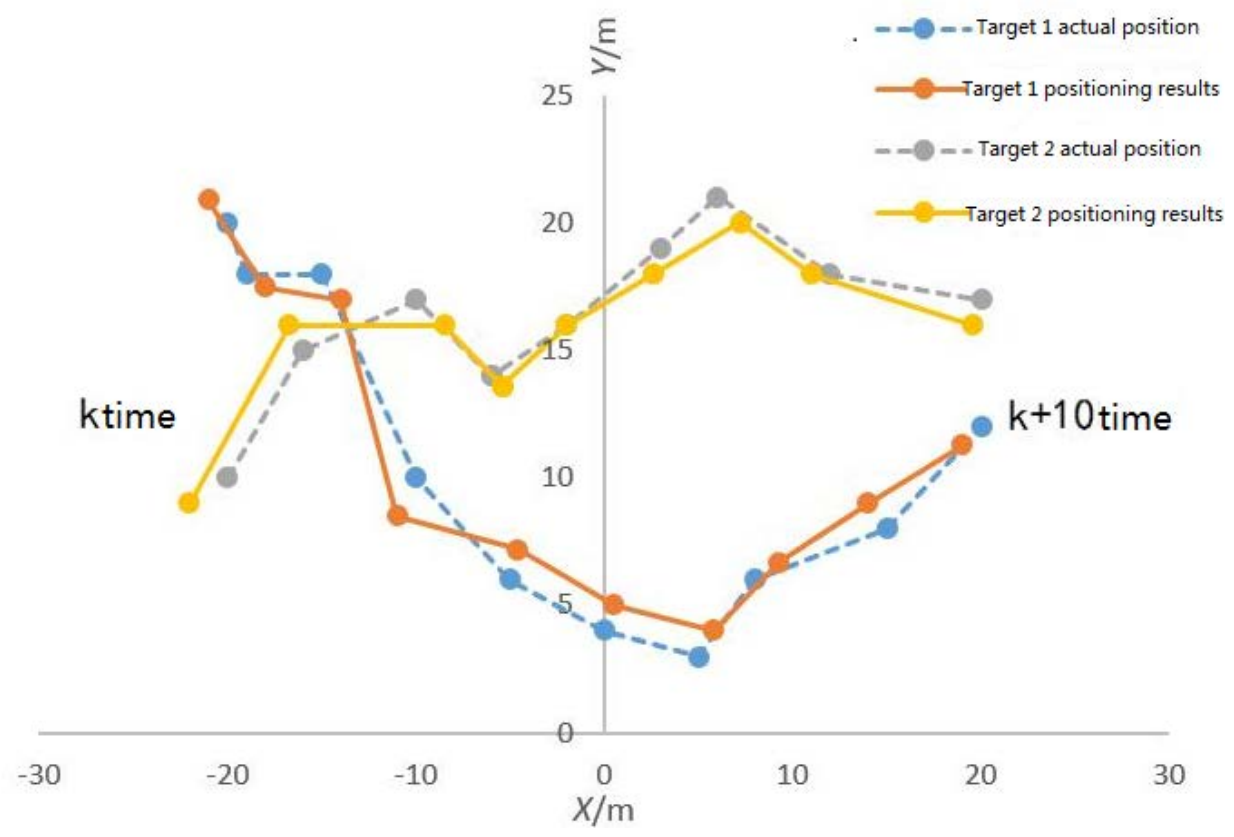

Fig.3 Positioning results of multi-targets at a given interval 
According to the cumulative probability distribution of location error in Fig. 4, we can see that the error of location method in this paper is within $0.8 \mathrm{~m}$ under the probability of nearly $85 \%$, which is better than the traditional RSSI and infrared location methods, while the cumulative probability distribution tends to 1 . The error of the algorithm proposed in this paper is obviously bigger than the traditional two methods. These larger errors all happen in the boundary of the monitoring area, because the monitoring nodes of the sensor network have low intersection density. This also increases the average error of the method, but it can be solved by expanding the monitoring range of the sensor network and improving the intersection density of the monitoring range of the nodes, that is to say, the monitoring range of the sensor network is larger than the actual monitoring boundary.

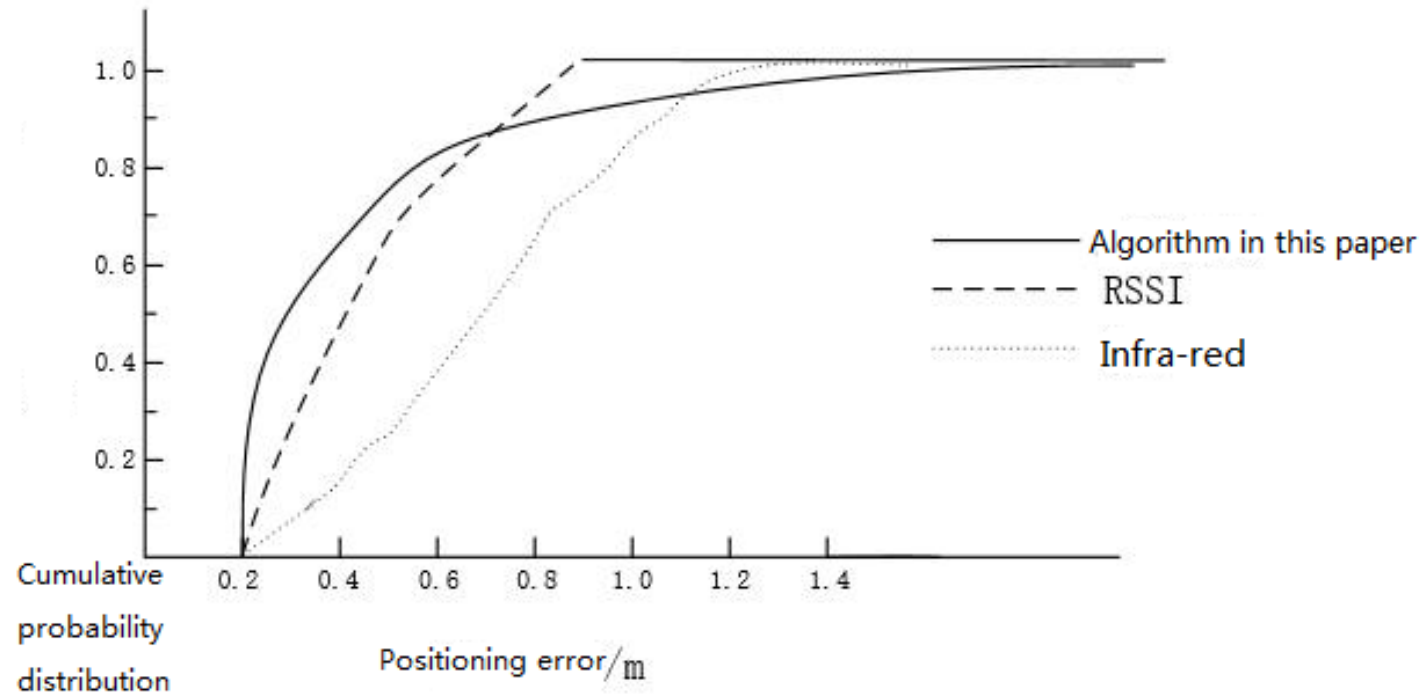

Fig.4 Cumulative probability distribution of location deviation

\section{Conclusion}

In this paper, an outdoor adaptive location method based on clustering and RSSI is proposed for the passive multi target positioning technology in wireless sensor networks. Clustering is applied to achieve clustering of targets. Then, RSSI distance, infrared ranging and weighted multilateral centroid algorithm are applied to calculate the final coordinates of passive targets, thus reducing the error of passive multi-target localization. The simulation results show that the location error is less than $0.8 \mathrm{~m}$ under the probability of $85 \%$, and the larger error of the regional boundary can be solved by expanding the monitoring area and the density of the boundary nodes. The location results of multi-target are consistent with the trend of actual location and track. Therefore, the algorithm in this paper can meet the location requirements of passive multi-target in the field environment.

\section{Acknowledgement}

This paper is grateful for the support of three fund projects: Fujian Provincial Natural Science Foundation (2015J05010); Longyan University youth climbing project (LQ2016004); college students' innovation and entrepreneurship training program.

\section{References}

[1] Shen X, Yang J. Node Self-Localization Algorithm Based on RSSI in Wireless Sensor Networks Outdoor[C]// International Conference on Intelligent System Design and Engineering Application. IEEE Computer Society, 2010:1010-1012.

[2] Dong Q, Xu X. A Novel Weighted Centroid Localization Algorithm Based on RSSI for an Outdoor Environment [J]. Journal of Communications, 2014, 9(3):279-285.

[3] Pricone M, Caracaş A. A heterogeneous RSSI-based localization system for indoor and outdoor 
sports activities[C]// Wireless Communications and Mobile Computing Conference. IEEE, 2014:274-280.

[4] Adewumi O G, Djouani K, Kurien A M. RSSI based indoor and outdoor distance estimation for localization in WSN[C]// IEEE International Conference on Industrial Technology. IEEE, 2013:1534-1539.

[5] Guo W, Deng Y, Yang Y, et al. A novel outdoor localization with cellular topology in opportunistic networks[C]// International Conference on Automatic Control and Artificial Intelligence. IET, 2013:239-242.

[6] Chen Y S, Chin T L, Huang Y C. Collaborative localization in Wireless Sensor Networks based on dependable RSSI[C]// International Symposium on Wireless Personal Multimedia Communications. IEEE, 2012:341-347.

[7] El-Din R A Z, Rizk M. Accurate indoor localization based on RSSI with adaptive environmental parameters in wireless sensor networks[C]// First International Conference on Innovative Engineering Systems. IEEE, 2013:177-183.

[8] Gharghan S K, Nordin R, Ismail M, et al. Accurate Wireless Sensor Localization Technique Based on Hybrid PSO-ANN Algorithm for Indoor and Outdoor Track Cycling[J]. IEEE Sensors Journal, 2015, 16(2):529-541.

[9] Gani M O, Obrien C, Ahamed S I, et al. RSSI Based Indoor Localization for Smartphone Using Fixed and Mobile Wireless Node[C]// Computer Software and Applications Conference. IEEE, 2013:110-117.

[10] Agroudy N E, Joram N, Ellinger F. Low power RSSI outdoor localization system[C]// Ph.d. Research in Microelectronics and Electronics. IEEE, 2016:1-4.

[11] Kuhnert L, Meier B, Kuhnert K D. Probabilistic approach to self-localization for autonomous mobile outdoor robotics based on hybrid map knowledge[C]// International IEEE Conference on Intelligent Transportation Systems. IEEE, 2013:1791-1798.

[12] Peng H, Liu S, Wang J, et al. An image matching method based on BoVW model for visual self-localization[C]// Control Conference. IEEE, 2014:8513-8517. 